\title{
DINÁMICAS SUCESIONAL PRIMARIA NATURAL Y SECUNDARIA ANTROPOGÉNICA DE LA VEGETACIÓN DEL HUMEDAL “CIÉNAGAS DEL NAME" (CHILE CENTRAL): UN MODELO CONCEPTUAL
}

\author{
PRIMARY NATURAL AND SECONDARY ANTHROPOGENIC \\ SUCCESSIONAL DYNAMICS OF WETLAND VEGETATION IN \\ “CIÉNAGAS DEL NAME" (CENTRAL CHILE): A CONCEPTUAL \\ MODEL
}

\author{
Carlos Ramírez ${ }^{1 *}$, José Miguel Fariña ${ }^{1}$, Domingo Contreras ${ }^{2}$, Cristina San Martín ${ }^{3}$, Andrés Camaño ${ }^{4}$, \\ Miguel Álvarez ${ }^{5}$, Osvaldo Vidal ${ }^{6}$, José Luis Solís ${ }^{3}$, Yessica Pérez ${ }^{3}$ y Oliver Valdivia ${ }^{7}$. \\ ${ }^{1}$ Departamento de Ecología, Facultad de Ciencias Biológicas, Universidad Católica de Chile, Santiago, \\ Chile. \\ 2 Facultad de Ciencias Básicas, Universidad Católica del Maule, Talca, Chile. \\ ${ }^{3}$ Instituto de Ciencias de la Tierra, Facultad de Ciencias, Universidad Austral de Chile, Valdivia, Chile. \\ ${ }^{4}$ Environmental Resources Management (ERM), Santiago, Chile. \\ ${ }^{5}$ INRES-Vegetationsökologie, Rheinische Friedrich-Wilhelms-Universität, Bonn, Alemania. \\ ${ }^{6}$ Instituto de la Patagonia, Universidad de Magallanes, Punta Arenas, Chile. \\ 7 Escuela de Agronomía, Universidad de Tarapacá, Arica, Chile. \\ * Autor para correspondencia E-mail: cramirez@uach.cl
}

\section{RESUMEN}

Se estudió la dinámica de la vegetación del humedal "Ciénagas del Name" ubicado en la provincia de Cauquenes, Región del Maule, Chile, incluyendo la vegetación acuática y palustre de la laguna, y la terrestre de zonas aledañas. Aplicando el índice de similitud de Bray-Curtis, el primer conglomerado reunió comunidades palustres y el segundo, terrestres y acuáticas, el análisis de ordenación permitió homologar los primeros ejes a gradientes de humedad y temperatura. El origen fitogeográfico de las especies permitió distinguir comunidades originales primarias con pocas especies introducidas, y secundarias y terciarias antropogénicas, con varias. Se construyó un modelo conceptual de la posible dinámica de la vegetación que distingue, una sucesión natural de relleno de la laguna y otra antropogénica correspondiente a la degradación del bosque esclerófilo primitivo mediante tala, pastoreo, cultivo y ruderalización, que permite además establecer el curso seguido por la sucesión en ambos casos. La sucesión acuática correspondería a un hidrosere natural que provoca el relleno de la laguna comenzando primero con comunidades acuáticas sumergidas, luego natantes, para llegar a un totoral que puede terminar en un bosque pantanoso nativo, pero actualmente, esta serie acuática se detiene en la etapa de totora (Schoenoplectus californicus) la que a su vez puede ser reemplazada por comunidades pratenses antropogénicas húmedas. La serie de degradación terrestre transforma el bosque esclerófilo en un matorral esclerófilo, que por pastoreo termina en una etapa permanente de espinal con Acacia caven, el que puede invadir las praderas húmedas, conectando ambas series.

Palabras clave: humedales, vegetación, dinámica, hidrosere, degradación antrópica. 


\begin{abstract}
The dynamics of wetland vegetation in "Laguna del Name", Cauquenes province, Maule Region, Chile was studied, including aquatic and swamp vegetation as well as surrounding terrestrial areas. By applying the Bray-Curtis similarity index, the first cluster put together the swamp communities, while the second cluster corresponded to terrestrial and aquatic communities; this ordination analysis was used to make equal the first two axes to temperature and moisture gradients. The plant geographical origin of the species allowed distinguishing primary native communities with few introduced species, as well as secondary and tertiary anthropogenic communities with several introduced species. A conceptual model of the potential vegetation dynamics was developed, identifying a natural succession (filling of the lagoon) and an anthropogenic succession, which corresponds to the degradation of the primitive sclerophyllous forest through logging, grazing, farming and ruderalization; this allowed determining the course followed in both cases. The aquatic succession may correspond to a natural hydrosere that would cause the filling of the lagoon, starting with submerged aquatic species, followed by floating leaf communities and leading to a reed bed that may end up in a native swamp forest. Today, this aquatic series stops at the reed-bed stage with Schoenoplectus californicus, which in turn can be replaced by anthropogenic wet meadow/pasture communities. The succession of land degradation transforms sclerophyllous native forest into a grazing "espinal" with Acacia caven, which can permanently invade the wet meadows, connecting both series.
\end{abstract}

Key words: wetland, vegetation, dynamic, hydrosere, anthropic degradation.

\section{INTRODUCCIÓN}

El paisaje vegetal de Chile Central se presenta muy diversificado con restos de bosques esclerófilos originales (comunidades primarias), con matorrales, espinales, praderas y cultivos (comunidades secundarias) que tienen origen antropogénico y cuya estructura depende del manejo practicado al suelo (San Martín y Donoso, 1996). Cuando el suelo degradado de las comunidades secundarias, alterado por sobrepastoreo y reiterados cultivos, es abandonado, se cubre con comunidades ruderales (terciarias), volviéndose improductivo. Estas comunidades terciarias cumplen una importante función ecológica al evitar la erosión y recuperar a largo plazo el suelo, especialmente cuando están formadas por leguminosas (Ramírez et al., 1988).

En dichos mosaicos de vegetación es muy importante conocer la relación de origen entre las diversas comunidades (asociaciones) vegetales, para reconstruir teóricamente primero e intentar recuperar prácticamente después, la vegetación original (Ramírez et al., 2012). También este análisis del origen permite conocer el curso seguido por las sucesiones primarias y secundarias, lo que facilita predecir los cambios de vegetación que se producirán en un lugar con un determinado tipo de manejo. Ambos conocimientos son importantes herramientas para la planificación del uso del suelo y por ende, del paisaje (Ellies y Ramírez, 1994; Peña-Cortés et al., 2006).

El estudio de la vegetación (comunidades vegetales) presente en un determinado lugar es básico para realizar otros como los de dinámica vegetacional. Para ello es muy apropiada la metodología fitosociológica de la Escuela Zürich-Montpellier (Mueller-Dombois y Ellenberg, 1974), que permite clasificar la vegetación mediante relevamientos en terreno, utilizando especies características y diferenciales que caracterizan y diferencian las grandes unidades de vegetación (alianza, orden, clase) las primeras, y las menores (asociaciones, sub-asociaciones, variantes), las segundas (Dengler et al., 2008).

Establecer las relaciones de origen de cada comunidad es un problema complicado porque se produce en un lapso temporal, que normalmente supera la duración de una vida humana. Por ello es necesario reemplazar el tiempo por otras variables, como por ejemplo el espacio o la composición florística. Esta última es muy importante para determinar el carácter primario, secundario o terciario de cada comunidad al conocer la proporción entre especies nativas e introducidas en cada una. La mayor cantidad de especies alóctonas se presenta generalmente en las comunidades secundarias de una serie degradativa de la vegetación, ya que en las terciarias, suelen aparecer nuevamente algunas especies nativas (Ramírez et al., 2012). En los últimos años ha dado muy buen resultado la utilización de métodos estadísticos multivariados que al comparar cada especie y asociación vegetal con cada una de las otras en una tabla de vegetación, revive en cierta forma, la relación de origen entre las últimas, y las ubican en el lugar correspondiente de la sucesión (Ortuño et al., 2006). La presencia de malezas indicadoras y análisis físico-químicos del suelo permiten 
determinar el tipo de manejo realizado en cada comunidad (San Martín et al., 2014).

El presente estudio pretende conocer la dinámica del mosaico vegetacional acuático, palustre y terrestre del humedal de las "Ciénagas del

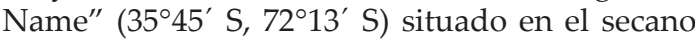
costero de la Región del Maule (Chile) en la provincia de Cauquenes, a unos $20 \mathrm{~km}$ de la ciudad homónima. En los últimos años este humedal ha sido objeto de varios estudios, entre ellos, Arellano et al. (2013) que estudiaron su rol en el secuestro de carbono, Ramírez et al. (2014a) la flora, y Ramírez et al. (2014b) la vegetación. En este último trabajo se describieron las 13 comunidades (asociaciones) vegetales objeto de este estudio (Tabla 1).

Cómo hipótesis se planteó que en esas comunidades deberían estar representadas como mínimo dos series sucesionales, una primaria que provocaría el relleno de las lagunas del humedal, y otra secundaria, correspondiente a la degradación de la vegetación original terrestre. Este estudio servirá de base para compatibilizar la actividad silvoagropecuaria de los terrenos adyacentes al humedal y la conservación del mismo, que es una preocupación actual de los vecinos y de los organismos estatales encargados de la protección de los ecosistemas.

\section{MATERIALES Y MÉTODOS}

El material de este estudio corresponde a una tabla de vegetación resumida formada por 13 columnas o asociaciones vegetales presentes en las
Ciénagas del Name y 73 filas que contienen otras tantas especies vegetales presentes con distinta frecuencia y cobertura en las comunidades indicadas en la Tabla 2, construida con los censos de Ramírez et al. (2014b). Cada columna muestra la cobertura promedio obtenida a partir de un mínimo de 3 censos de vegetación en cada comunidad. La cobertura está expresada en porcentaje y corresponde al promedio elevado a la unidad superior cuando se presenta fraccionado. Los censos de las comunidades boscosas nativas fueron tomados de la literatura, debido a que los rodales de ellas se encuentran actualmente muy fraccionados, alterados y degradados, siendo imposible inventariarlos fitosociológicamente (Amigo y Flores-Toro, 2013). Los nombres científicos latinizados de las especies tienen dos palabras con diferentes terminaciones y los de las comunidades, dos o más palabras pero con la terminación -etum en una de ellas.

De la primera columna de la Tabla 2 se tomó la lista de especies para analizar su presencia en las diferentes comunidades, considerando el número de especies por cada una, su frecuencia en la tabla y el origen fitogeográfico (nativa o introducida). También se determinaron las formas de vida de las especies utilizando la clave de Mueller-Dombois y Ellenberg (1974). Se consideraron sólo las 5 formas de vida principales: fanerófitos (árboles, arbustos, lianas, epífitas y parásitas), caméfitos (subarbustos, plantas en cojin o pulviniformes y hierbas altas erguidas), hemicriptófitos (hierbas perennes), criptófitos o hierbas perennes con órganos subterráneos de reserva que sobreviven en

Tabla 1. Unidades de la vegetación acuática y terrestre del humedal "Ciénagas del Name" y zonas aledañas. Las abreviaturas de la primera columna se utilizan en las siguientes tablas y figuras.

Table 1. Units of aquatic and terrestrial vegetation of the wetland "Ciénagas del Name" and surrounding areas. The abbreviations of the first column is used in the following tables and figures.

\begin{tabular}{cllc}
\hline Abreviatura & Asociación vegetal & Comunidad & Especies \\
\hline $\mathrm{BM}$ & Temo-Myrceugenietum & Bosque de temo-pitra & 8 \\
$\mathrm{QL}$ & Quillajo-Lithraetum & Bosque de litre-quillay & 9 \\
$\mathrm{CT}$ & Cestro-Trevoetum & Espinal & 17 \\
$\mathrm{Pi}$ & Plantación de pino & Bosque de pino & 15 \\
$\mathrm{RC}$ & Rubo-Cestretum parqui & Matorral de zarzamora & 12 \\
$\mathrm{Sc}$ & Scirpetum californiae & Pantano de totora & 12 \\
$\mathrm{~GB}$ & Gutierrezo-Baccharietum & Matorral de huatro & 11 \\
$\mathrm{MJ}$ & Mentho-Juncetum & Pradera de poleo & 10 \\
$\mathrm{Jp}$ & Juncetum procerii & Pradera de Junquillo & 8 \\
$\mathrm{LA}$ & Lemno-Azolletum & Flotante libre de flor del pato & 6 \\
$\mathrm{Pl}$ & Potamogetetum lucentis & Sumergida de huiro verde & 6 \\
$\mathrm{PL}$ & Polygono-Ludwigietum & Natante de clavito de agua & 5 \\
$\mathrm{UC}$ & Utriculario-Ceratophylletum & Sumergida de ceratofilo & 4 \\
\hline
\end{tabular}


Tabla 2. Tabla resumen de la vegetación de las Ciénagas del Name y zonas aledañas, ordenada por la frecuencia (Fr.) de las especies. El significado de las abreviaturas de las asociaciones vegetales se encuentra en la Tabla 1. Los números indican porcentaje promedio de cobertura subidos a la unidad superior.

Table. 2. Summary table of the vegetation "Ciénagas del Name" and surrounding areas, ordered by frequency (Fr.) of the species. The meaning of abbreviations of plant associations is in Table 1. The numbers indicate cover average uploaded to the next whole percentage.

\begin{tabular}{|c|c|c|c|c|c|c|c|c|c|c|c|c|c|c|}
\hline Especies / Asociaciones: & BM & I QL & Pi & CT & RC & GB & MJ & $\mathrm{Jp}$ & Sc & PL & $\mathrm{UC}$ & LA & Pl & Fr. \\
\hline Acacia caven & & 3 & 3 & 40 & 1 & 2 & 1 & & & & & & & 6 \\
\hline Leontodon saxatilis & & & & 80 & & 1 & 10 & 1 & & & & & & 4 \\
\hline Mentha pulegium & & & & 15 & & & 50 & 60 & 20 & & & & & 4 \\
\hline Juncus procerus & 5 & & & 1 & & & 1 & 50 & & & & & & 4 \\
\hline Ludwigia peploides & & & & & & & & & 1 & 40 & 1 & & 5 & 4 \\
\hline Otholobium glandulosum & 5 & 1 & & 1 & 1 & & & & & & & & & 4 \\
\hline Rosa rubiginosa & 1 & 1 & & & 1 & 1 & & & & & & & & 4 \\
\hline Eleocharis pachycarpa & & & & & & & & 10 & 20 & 30 & & & & 3 \\
\hline Peumus boldus & 5 & 5 & 1 & & & & & & & & & & & 3 \\
\hline Phyla nodiflora & & & & 1 & & & 1 & 1 & & & & & & 3 \\
\hline Myriophyllum aquaticum & & & & & & & & & & 5 & 1 & 1 & & 3 \\
\hline Utricularia gibba & & & & & & & & & & & 5 & 1 & 1 & 3 \\
\hline Quillaja saponaria & & 45 & 5 & & & & & & & & & & & 2 \\
\hline Lithrea caustica & & 15 & 3 & & & & & & & & & & & 2 \\
\hline Juncus microcephalus & & & & & & & 20 & 1 & & & & & & 2 \\
\hline Schoenoplectus californicus & & & & & & & & & 60 & 1 & & & & 2 \\
\hline Polygonum hydropiperoides & & & & & & & & 1 & 20 & & & & 2 & \\
\hline Azolla filiculoides & & & & & & & & & & & & 25 & 1 & 2 \\
\hline Ricciocarpus natans & & & & & & & & & & & & 10 & 1 & 2 \\
\hline Myrceugenia obtusa & 10 & 5 & & & & & & & & & & & & 2 \\
\hline Colliguaja odorifera & 1 & 1 & & & & & & & & & & & & 2 \\
\hline Maytenus boaria & & 5 & 1 & & & & & & & & & & & 2 \\
\hline Gochnatia foliosa & & & 1 & 1 & & & & & & & & & & 2 \\
\hline Vulpia bromoides & & & 1 & 1 & & & & & & & & & & 2 \\
\hline Vitis vinifera & & & 1 & & 1 & & & & & & & & & 2 \\
\hline Trifolium dubium & & & & 1 & & & & 1 & & & & & & 2 \\
\hline Chamaemelum mixtum & & & & 1 & & 30 & & & & & & & & 2 \\
\hline Avena barbata & & & & & 1 & 1 & & & & & & & & 2 \\
\hline Gratiola peruviana & & & & & & & & 5 & 1 & & & & & 2 \\
\hline Myrceugenia exsucca & 40 & & & & & & & & & & & & & 1 \\
\hline Pinus radiata & & & 70 & & & & & & & & & & & 1 \\
\hline Rubus ulmifolius & & & & & 95 & & & & & & & & & 1 \\
\hline Baccharis linearis & & & & & & 55 & & & & & & & & 1 \\
\hline Ceratophyllum chilense & & & & & & & & & & & 99 & & & 1 \\
\hline Lemna minima & & & & & & & & & & & & 60 & & 1 \\
\hline Potamogeton lucens & & & & & & & & & & & & & 95 & 1 \\
\hline Salix humboldtiana & 10 & & & & & & & & & & & & & 1 \\
\hline Plantago lanceolata & & & 1 & & & & & & & & & & & 1 \\
\hline Digitalis purpurea & & & 1 & & & & & & & & & & & 1 \\
\hline Dioscorea bryoniifolia & & & 1 & & & & & & & & & & & 1 \\
\hline Trevoa trinervis & & & 1 & & & & & & & & & & & 1 \\
\hline Kageneckia oblonga & & & 1 & & & & & & & & & & & 1 \\
\hline Nasella chilensis & & & 1 & & & & & & & & & & & 1 \\
\hline Agrostis stolonifera & & & & 1 & & & & & & & & & & 1 \\
\hline Briza minima & & & & 1 & & & & & & & & & & 1 \\
\hline Bromus hordeaceus & & & & 1 & & & & & & & & & & 1 \\
\hline Cyperus reflexus & & & & 1 & & & & & & & & & & 1 \\
\hline Hordeum chilense & & & & 1 & & & & & & & & & & 1 \\
\hline
\end{tabular}


Linum usitattisimum

Lotus corniculatus

1

Muehlenbeckia hastulata

Carduus pycnocephalus

Cuscuta chilensis

Salix viminalis

Solanum nigrum

Medicago polymorpha

Baccharis concava

Aira caryophyllea

Silena gallica

Erodium cicutarium

Cirsium vulgare

Juncus imbricatus

Aster vahlii

Hedyotis salzmannii

Nierenbergia repens

Cyperus conceptionis

Holcus lanatus

Hypochaeris radicata

Lactuca serriola

Rumex acetosella

Senecio aquaticus

Wolffiella oblonga

Potamogeton pusillus

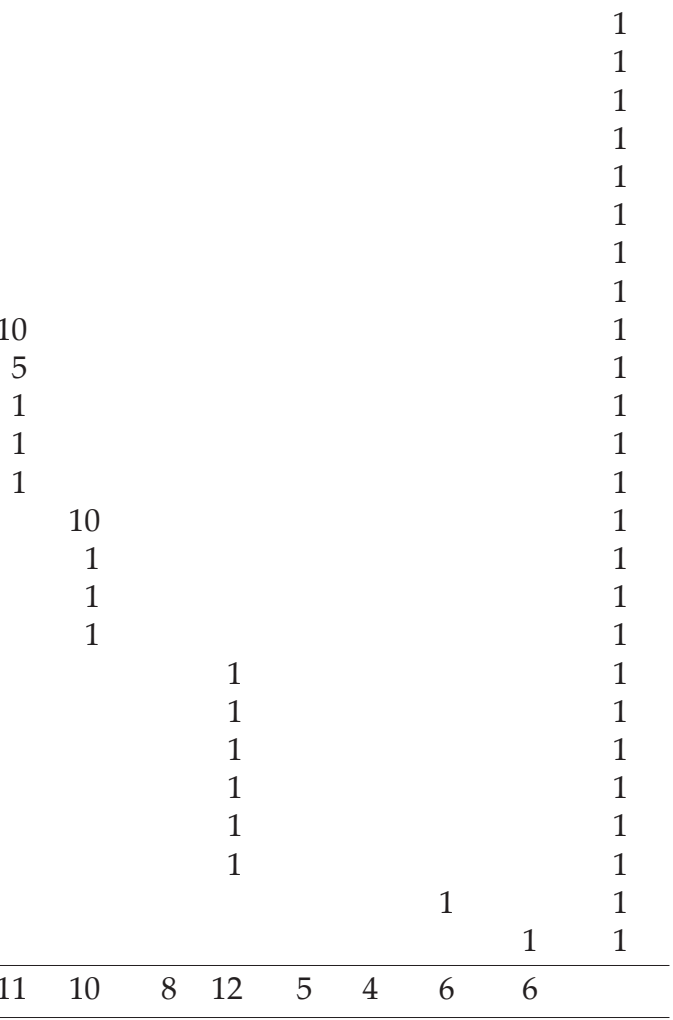

Total (73 especies):

$\begin{array}{lllll}8 & 9 & 15 & 17 & 12\end{array}$

invierno (geófitos terrestres) que incluyen además helófitos palustres e hidrófitos acuáticos, y por último, los terófitos (hierbas anuales y bianuales). Con estas formas de vida se confeccionaron espectros biológicos y se estableció la estratificación de las diferentes comunidades.

Las asociaciones vegetales de la tabla fitosociológica promedio fueron comparadas en su composición florística con el índice de disimilitud de Bray-Curtis que se mueve entre 0 y 1 , el primero indica completa similitud y el segundo, ausencia de especies en común, es decir, completa disimilitud (Bray y Curtis, 1957). Para la clasificación de las comunidades vegetales se utilizó la matriz de distancia aplicando una agrupación de clusters (grupos) jerárquicos según el algoritmo de Ward (Gautreau y Lezama, 2009). La misma matriz de disimilitud fue usada para realizar un análisis NMDS (non-metric multidimensional scaling) restringiendo la ordenación a dos dimensiones en las cuales se ordenaron las especies y los centroides de las comunidades (Clarke, 1993; Legendre y Legendre, 1998). El estadístico resultante es un valor denominado "Stress", el cual indica como la configuración de la gráfica representa las distancias entre los sitios (Roeland y Coe, 2005). Los análisis multivariados se realizaron usando los paquetes estadísticos BiodiversityR (Roeland y Coe, 2005) y MASS v. 7.3 (Venables y Ripley, 2002) mediante la versión R Studio 2.15.1 (R De- velopment Core Team, 2012). En la ordenación de las especies se relacionaron los dos ejes utilizados con gradientes ecológicos, lo que permitió comprender la distribución y los requerimientos de sitio de las asociaciones vegetales en el mismo arreglo.

Se construyeron diagramas de flujo con los resultados de los análisis multivariados, con la literatura disponible y con observaciones de terreno, que permitieron identificar las posibles series de evolución o degradación de las asociaciones vegetales para conocer la dinámica de la vegetación y sus posibles relaciones de origen. Finalmente, se construyó un modelo conceptual simplificado, que permite entender y conocer la compleja dinámica vegetacional que ha tenido lugar en épocas pasadas en las Ciénagas del Name, hasta llegar a la situación actual, y los posibles cambios que podrían acontecer en el futuro de acuerdo a los diferentes manejos del suelo (Greca y Moreira, 1998).

\section{RESULTADOS}

\section{Flora}

Las 73 especies de la tabla fitosociológica inicial se distribuyen en las 11 asociaciones o comunidades vegetales que se presentan ordenadas desde la tierra hacia el agua (Tabla 2). La mayor cantidad de especies (17) se presentó en el espinal, estadio degradado de la asociación secunda- 
ria Cestro-Trevoetum trinervis (matorral esclerófilo) y la menor (4) se presentó en la asociación de ceratofilo (Utriculario-Ceratophylletum chilensis), lo que está de acuerdo con el carácter extremo de su hábitat acuático sumergido.

El número de especies fue menor en ambos extremos, es decir, en la vegetación boscosa y en las comunidades acuáticas, ambas primarias (Fig. 1). La escasez de especies en las comunidades boscosas primarias no es real, y se debe más que nada a que se censaron pocos rodales y además, alterados. Las comunidades intermedias de la zona ecotonal y la plantación de pino insigne (Pinus radiata D. Don) se ubican con valores altos en la mitad del diagrama, en este caso, la comunidad pantanosa de totora situada entre las praderas húmedas y la comunidad natante de clavito de agua (Polygono-Ludwigietum peploidis) compartiendo especies con ambas.

En la última columna de la Tabla 2 se muestra la frecuencia de las especies, es decir, la presencia de cada una en las asociaciones. Sólo el espino maulino (Acacia caven) se presentó en 6 comunidades con alta frecuencia, le siguen: Leontodon saxatilis, Mentha pulegium, Rosa rubiginosa, Juncus procerus, Ludwigia peloides y Otholobium glandulosum, presentes en 4 comunidades diferentes cada una. Las tres primeras son introducidas y las tres restantes, nativas. Cinco especies están presentes en tres comunidades diferentes: Eleocharis pachycarpa, Peumus boldus, Phyla nodiflora, Myriophyllum aquaticum y Utricularia gibba, todas ellas nativas, la mayoría de lugares húmedos y anegados y sólo una, el boldo, de lugares terrestres secos. Por último, hay 17 especies que aparecen en dos comunidades diferentes, y 44 en una sola (Fig. 2).

Como era de esperar, en los bosques primarios las especies nativas son más abundantes (Tabla 3) pero aunque en la plantación de pino domina Pinus radiata, en el sotobosque aparecen muchas especies leñosas y herbáceas nativas. En los dos matorrales secundarios y en el espinal dominan las especies introducidas, lo que también sucede en el totoral, el resto de las comunidades de praderas húmedas, pantanosas y acuáticas presentan mayor cantidad de especies nativas, incluso el Lemno-Azolletum filiculoidis y el Utriculario-Ceratophylletum chilensis, carecen de especies alóctonas (Fig. 3).

\section{Espectros biológicos}

En los bosques y matorrales dominan las especies leñosas (fanerófitos), pero desaparecen en las comunidades palustres y acuáticas (Tabla 3). Los hemicriptófitos (hierbas perennes) están presentes en el $61,54 \%$ de las comunidades, siendo la forma de vida más frecuente en la vegetación de las Ciénagas del Name. Los criptófitos (hierbas acuáticas y palustres) están presentes sólo en comunidades anegadas, en cambio, los geófitos (hierbas terrestres con órganos subterráneos de reserva) y los terófitos (hierbas anuales y bianuales) abundan en comunidades terrestres pratenses (Fig. 4), siendo la mayoría malezas alóctonas.

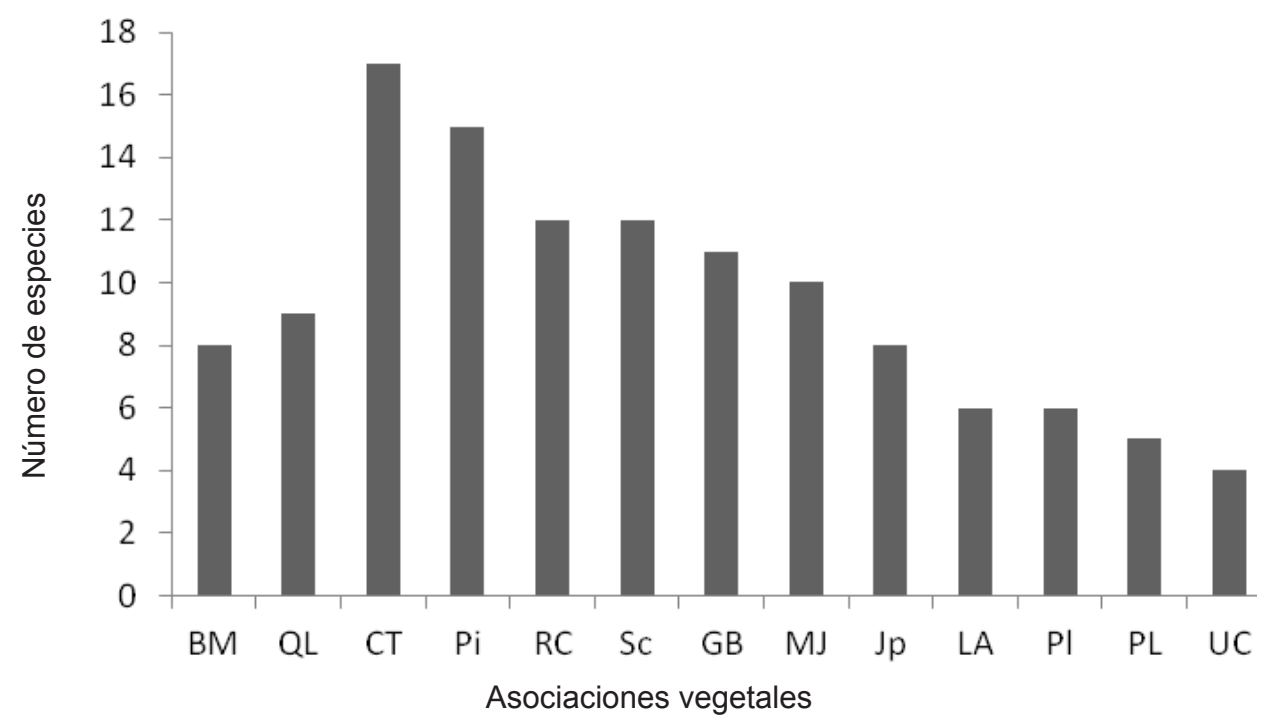

Fig. 1. Número de especies de cada asociación vegetal de la Tabla 2. El significado de las abreviaturas de las asociaciones vegetales se encuentra en la Tabla 1.

Fig. 1. Number of species of each plant association of the Table 2. The meaning of the abbreviations of plant associations found in Table 1. 


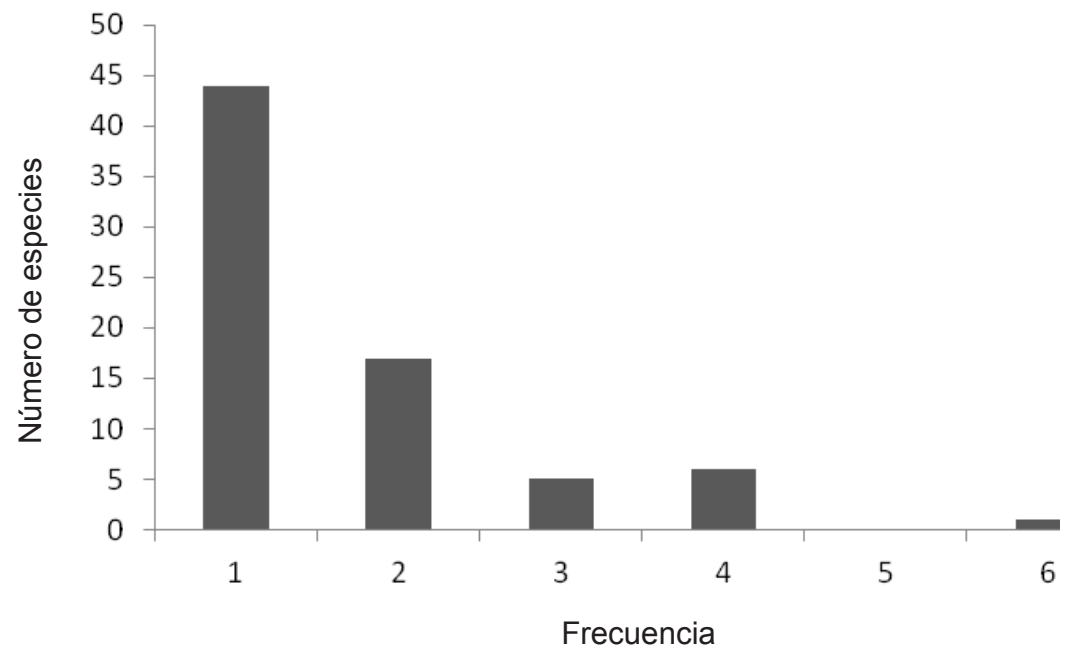

Fig. 2. Número de especies por tramo de frecuencia de la Tabla 2.

Fig. 2. Number of species per frequency segment of Table 2.

Tabla 3. Origen fitogeográfico y formas de vida de las especies y número de estratos por asociación vegetal en las asociaciones vegetales de las Ciénagas del Name y zonas aledañas. El significado de las abreviaturas de las asociaciones vegetales se encuentra en la Tabla 1.

Tabla 3. Phytogeographical origin and life forms of the species and number of strata in plant associations of "Ciénagas del Name" and surrounding areas. The meaning of abbreviations plant association is found in Table 1.


Origen fitogeográfico

Especies

Nativas

Introducidas

Total especies:

$\begin{array}{rrrrrrrrrrrrr}7 & 8 & 10 & 7 & 4 & 3 & 8 & 5 & 5 & 4 & 4 & 6 & 5 \\ 1 & 1 & 5 & 10 & 8 & 8 & 2 & 3 & 7 & 1 & 0 & 0 & 1 \\ \mathbf{8} & \mathbf{9} & \mathbf{1 5} & \mathbf{1 7} & \mathbf{1 2} & \mathbf{1 1} & \mathbf{1 0} & \mathbf{8} & \mathbf{1 2} & \mathbf{5} & \mathbf{4} & \mathbf{6} & \mathbf{6}\end{array}$

Formas de Vida

Fanerófitos

Caméfitos

Hemicriptófitos

Criptófitos

Terófitos

$\begin{array}{rrrrrrrrrrrrr}7 & 9 & 10 & 3 & 8 & 4 & 1 & 0 & 0 & 0 & 0 & 0 & 0 \\ 0 & 0 & 0 & 1 & 0 & 0 & 1 & 2 & 2 & 0 & 0 & 0 & 0 \\ 1 & 0 & 3 & 7 & 1 & 2 & 8 & 5 & 6 & 1 & 0 & 0 & 0 \\ 0 & 0 & 0 & 0 & 0 & 0 & 0 & 0 & 3 & 4 & 4 & 6 & 5 \\ 0 & 0 & 2 & 6 & 3 & 5 & 0 & 1 & 1 & 0 & 0 & 0 & 0 \\ \mathbf{2} & \mathbf{1} & \mathbf{3} & \mathbf{3} & \mathbf{3} & \mathbf{3} & \mathbf{3} & \mathbf{3} & \mathbf{4} & \mathbf{2} & \mathbf{1} & \mathbf{1} & \mathbf{1}\end{array}$

\section{Estratificación}

Arbóreo

Arbustivo

Herbáceo alto

Herbáceo bajo

Total estratos:

$\begin{array}{lllllllllllll}1 & 1 & 1 & 1 & 0 & 0 & 0 & 0 & 0 & 0 & 0 & 0 & 0 \\ 1 & 1 & 1 & 1 & 1 & 1 & 0 & 0 & 0 & 0 & 0 & 0 & 0 \\ 0 & 0 & 0 & 1 & 1 & 0 & 1 & 1 & 1 & 1 & 0 & 0 & 0 \\ 0 & 1 & 1 & 1 & 0 & 1 & 1 & 1 & 1 & 1 & 1 & 1 & 1 \\ \mathbf{2} & \mathbf{3} & \mathbf{3} & \mathbf{4} & \mathbf{2} & \mathbf{2} & \mathbf{2} & \mathbf{2} & \mathbf{2} & \mathbf{2} & \mathbf{1} & \mathbf{1} & \mathbf{1}\end{array}$

Este espectro biológico es muy heterogéneo, seguramente por el carácter azonal de la mayoría de las comunidades vegetales presentes en el lugar.

La estratificación de la vegetación fue máxima en el espinal, presentando estratos arbóreos, arbustivos, herbáceo alto y herbáceo bajo (Tabla 3). El bosque de temo-pitra y las comunidades arbustivas y pratenses presentaron dos estratos, mientras que las comunidades acuáticas sólo tienen uno, siendo las más simples en su estructura. 


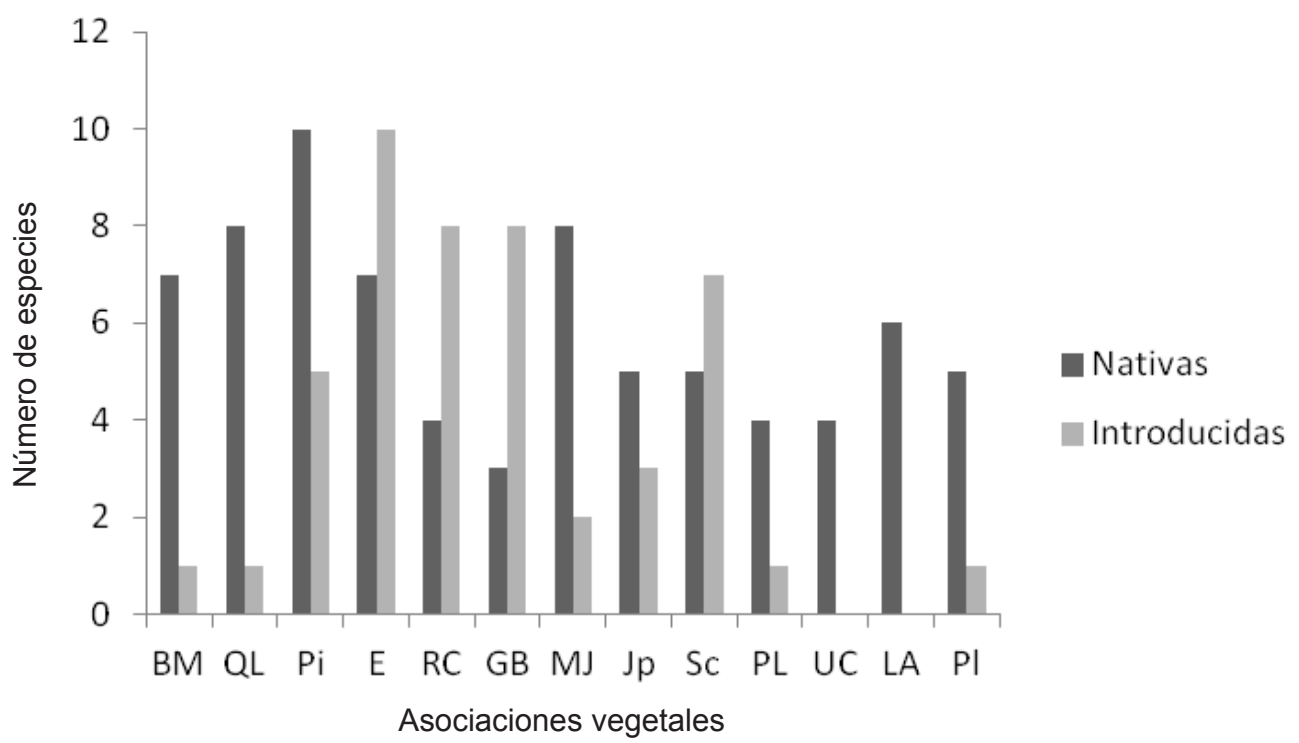

Fig. 3. Número de especies nativas e introducidas en cada asociación vegetal de la Tabla 2. El significado de las abreviaturas de las asociaciones vegetales se encuentra en la Tabla 1.

Fig. 3. Number of native and introduced species in each plant association of the Table 2 . The meaning of the abbreviations of plant associations found in Table 1.

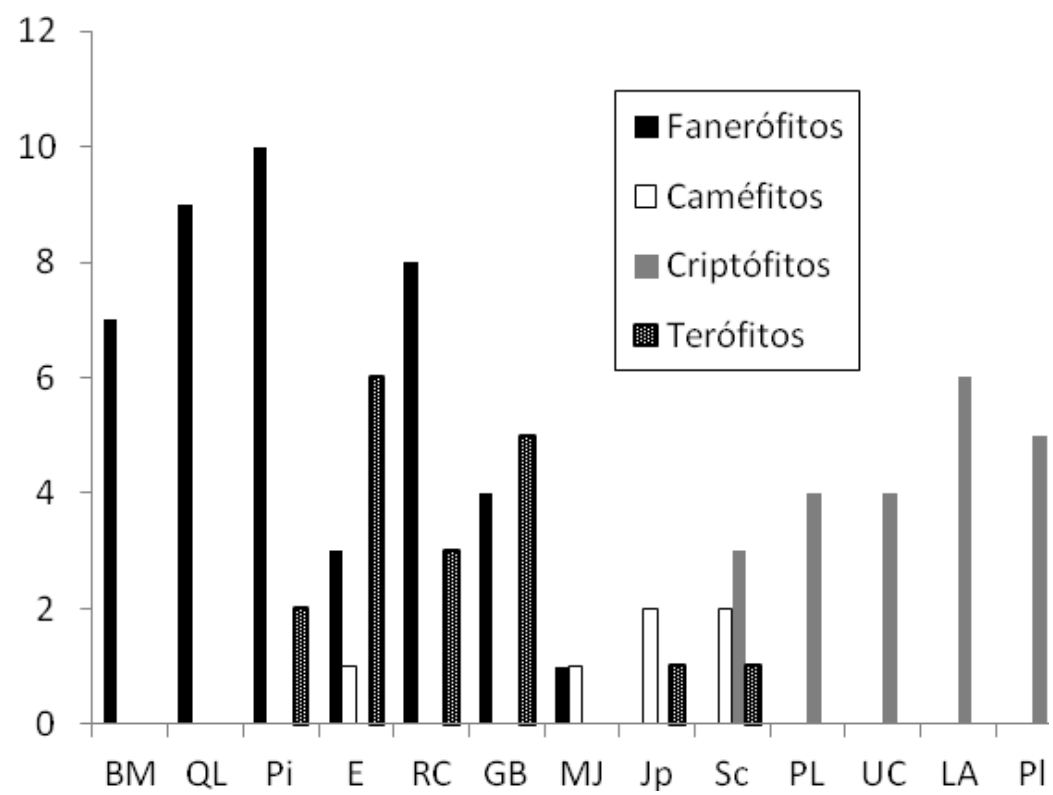

Fig. 4. Número de especies por cada forma de vida en las distintas asociaciones vegetales de la Tabla 2. El significado de las abreviaturas de las asociaciones vegetales se encuentra en la Tabla 1.

Fig. 4. Number of species per life form in the different plant associations of the Table 2 . The meaning of the abbreviations of plant associations found in Table 1.

Análisis de clasificación (conglomerados)

A la tabla fitosociológica se calculó el índice de disimilitud de Bray-Curtis entre asociaciones (Tabla 4). En ella se aprecia que las 4 comunidades vegetales acuáticas Polygono-Ludwigietum pe- ploidis, Utriculario-Ceratophylletum chilensis, Lemno-Azolletum filiculoidis y Potamogetetum lucentis, son totalmente disímiles a las asociaciones boscosas, arbustivas y pratenses húmedas, ya que no tienen especies en común con ellas. Las mayores 
Tabla 4. Matriz de disimilitud entre las asociaciones vegetales según índice de Bray-Curtis. El valor 0 indica total identidad entre las unidades, y el valor 1, unidades sin especies en común. El significado de las abreviaturas de las asociaciones se encuentra en la Tabla 1.

Table 4. Dissimilarity matrix between plant associations according to Bray-Curtis index. The value 0 indicates complete identity between the units and the value 1 , no species in common. The meaning of abbreviations plant associations found in Table 1.

\begin{tabular}{llllllllllllll}
\hline & BM & QL & Pi & CT & RC & GB & MJ & Jp & Sc & PL & UC & LA & Pl \\
\hline BM & 0 & 0,84 & 0,99 & 0,98 & 0,98 & 0,99 & 0,99 & 0,95 & 1 & 1 & 1 & 1 & 1 \\
QL & 0,84 & 0 & 0,85 & 0,97 & 0,97 & 0,97 & 0,99 & 1 & 1 & 1 & 1 & 1 & 1 \\
Pi & 0,99 & 0,85 & 0 & 0,96 & 0,98 & 0,98 & 0,99 & 1 & 1 & 1 & 1 & 1 & 1 \\
CT & 0,98 & 0,97 & 0,96 & 0 & 0,98 & 0,97 & 0,77 & 0,86 & 0,88 & 1 & 1 & 1 & 1 \\
RC & 0,98 & 0,97 & 0,98 & 0,98 & 0 & 0,97 & 0,99 & 1 & 1 & 1 & 1 & 1 & 1 \\
GB & 0,99 & 0,97 & 0,98 & 0,97 & 0,97 & 0 & 0,98 & 0,99 & 1 & 1 & 1 & 1 & 1 \\
MJ & 0,99 & 0,99 & 0,99 & 0,77 & 0,99 & 0,98 & 0 & 0,52 & 0,80 & 1 & 1 & 1 & 1 \\
Jp & 0,95 & 1 & 1 & 0,86 & 1 & 0,99 & 0,52 & 0 & 0,74 & 0,91 & 1 & 1 & 1 \\
Sc & 1 & 1 & 1 & 0,88 & 1 & 1 & 0,80 & 0,74 & 0 & 0,78 & 0,99 & 1 & 0,99 \\
PL & 1 & 1 & 1 & 1 & 1 & 1 & 1 & 0,91 & 0,78 & 0 & 0,98 & 0,99 & 0,95 \\
UC & 1 & 1 & 1 & 1 & 1 & 1 & 1 & 1 & 0,99 & 0,98 & 0 & 0,98 & 0,98 \\
LA & 1 & 1 & 1 & 1 & 1 & 1 & 1 & 1 & 1 & 0,99 & 0,98 & 0 & 0,97 \\
Pl & 1 & 1 & 1 & 1 & 1 & 1 & 1 & 1 & 0,99 & 0,95 & 0,98 & 0,97 & 0 \\
\hline
\end{tabular}

afinidades florísticas se presentan entre el Juncetum procerii y el Mentho-Juncetum microcephalii con un 0,52 de disimilitud; entre el Scirpetum californiae y el Juncetum procerii con un 0,74 de disimilitud; entre el Mentho-Juncetum microcephalii, y el estadio de espinal del Cestro-Trevoetum trinervis con un 0,77 de disimilitud; y entre el Scirpetum californiae y el Mentho-Juncetum microcephalii con un 0,80 de disimilitud. Estas comunidades de mayor similitud, corresponden a sintaxa intermedios en el gradiente vegetacional, que ponen en contacto las comunidades terrestres y acuáticas. La similitud entre la pradera de poleo Mentho-Juncetum micrephalii y el espinal demuestra que el último se introduce activamente hacia el pantano a medida que aumenta el relleno y baja la profundidad de la ribera. En este caso el espino (Acacia caven) coloniza las praderas húmedas actuando como una especie pionera en la recuperación de la primitiva vegetación terrestre boscosa.

El dendrograma de la Fig. 5 muestra las relaciones florísticas entre las distintas comunidades vegetales que conforman la vegetación de las Ciénagas del Name y sus alrededores. Se presentan dos grandes conglomerados, con 5 y 8 asociaciones vegetales, el primero y el segundo, respectivamente. El primer conglomerado reúne todas las comunidades vegetales intermedias de la zona ecotonal entre la vegetación acuática y la terrestre. En este grupo de la zona intermedia se presentan con alta similitud el Scirpetum californiae y el Polygono-Ludwigietum peploidis por un lado, y el Mentho-Juncetum microcephalii y el Juncetum procerii, por el otro.
En ambos casos se trata de pares de comunidades con alta relación de continuidad espacial en el gradiente. Lo interesante es que entre ambas, aunque formando parte del segundo subconglomerado, se presenta el estado de espinal del Cestro-Trevoetum trinervis, que como se dijo, va colonizando las praderas húmedas permitiendo el avance de la vegetación terrestre en el área de la vegetación acuática por relleno de la laguna (sucesión).

El segundo conglomerado presenta dos subconglomerados: el primero integrado por la plantación de pino y los bosques nativos esclerófilo (Quillajo-Lithraetum causticae) y pantanoso (Temo-Myrceugenietum exsuccae), estos último unidos con mayor similitud. Este conglomerado confirma el hecho de que el bosque de pino da lugar de vida a la flora de los bosques nativos, lo que podría permitir la regeneración del bosque primario, cuando se coseche la plantación de pino.

El segundo subconglomerado reúne con alta similitud dos matorrales Rubo-Cestretum parqui, secundario y Gutierrezo-Baccharietum linearis, terciario. Este último invade los sectores más degradados del espinal. Cerca de este conglomerado se forma un último que reúne las tres comunidades de vegetación acuática: Utriculario-Ceratophylletum chilensis, Lemno-Azolletum filiculoidis y Potamogetetum lucentis, la primera y la última sumergidas, $\mathrm{y}$ la del medio, flotante libre.

\section{Análisis de ordenación}

La Fig. 6 presenta la ordenación de las especies en los dos primeros ejes del análisis multi- 


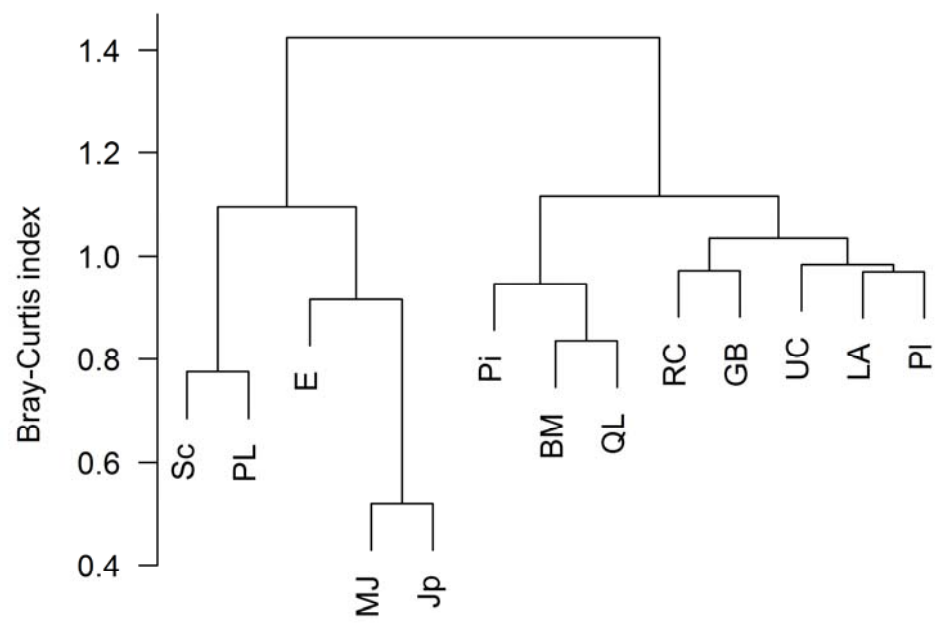

Fig. 5. Clasificación de las asociaciones vegetales de acuerdo al índice de disimilitud de Bray-Curtis. El significado de las abreviaturas de las asociaciones vegetales se encuentra en la Tabla 1.

Fig. 5. Classification of plant associations according to the dissimilarity index of Bray-Curtis. The meaning of the abbreviations of plant associations found in Table 1.

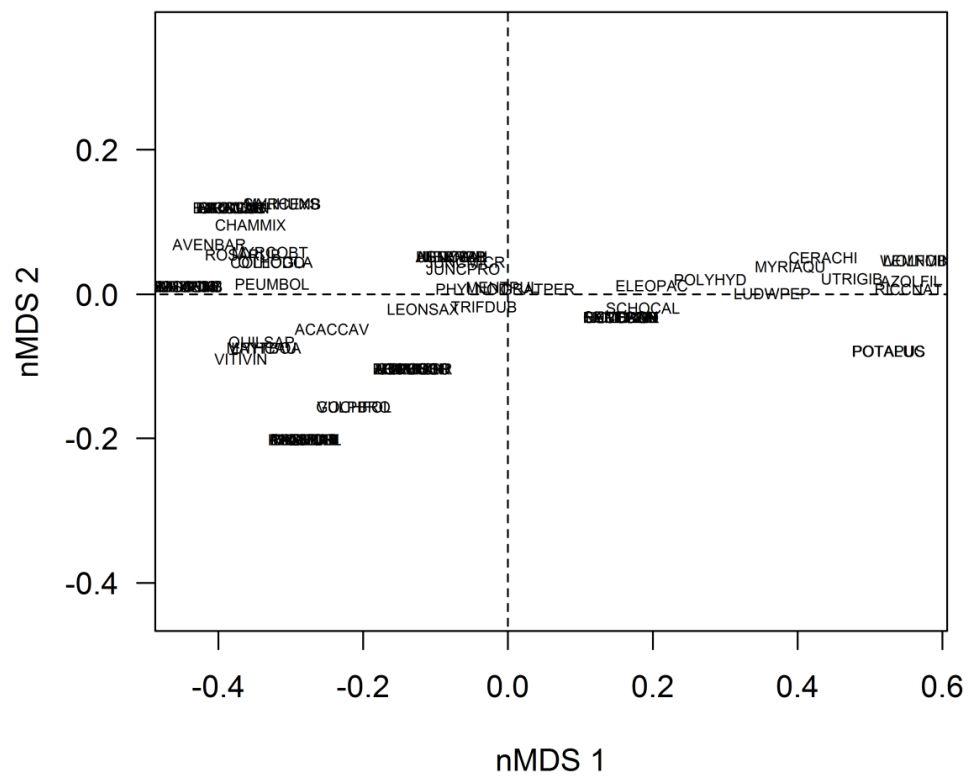

Fig. 6. Distribución de las especies de la Tabla 2 en los dos primeros ejes del análisis nMDS. Los nombres de las especies se abrevian con las 4 primeras letras del género y las tres primeras de la especie.

Fig. 6. Species distribution of the Table 2 in the first two axes of nMDS analysis. The species names are abbreviated with the first 4 letters of the genus and the first three of the species.

dimensional no métrico y escalado (NMDS) que las distribuye en un plano. El primer eje horizontal separa en su extremo izquierdo especies propias de los bosques esclerófilo y pantanoso y de la plantación de pino, incluso aquí se segregan claramente Vitis vinífera (vid) que sobrevive bajo de la plantación y Acacia caven que forma parte del bosque esclerófilo. Hacia la parte central del eje se ubican especies de matorrales secundarios, terciarios y de praderas húmedas. En el extremo derecho del eje se ubican especies palustres y acuáticas. Lo anterior permite relacionar este eje con un gradiente de humedad que aumentaría de izquierda a derecha.

Al observar la distribución de las especies a lo largo del segundo eje vertical se aprecia que ella 
es menor, concentrándose las especies en la parte media del eje y su mayor amplitud sólo se presenta en el extremo inferior izquierdo. Esto indica que la diferenciación por el factor que representaría este eje es menor, es decir, que ese factor presenta menos importancia para la vegetación de la laguna, lo que se confirma con la mayor amplitud que adquieren en el las especies de los bosques nativos. Por la disposición de las especies en el lado izquierdo se puede relacionar dicho eje con una gradiente de temperatura, que aumentaría de arriba hacia abajo. Lo anterior justifica el hecho de que las especies acuáticas y palustres muestren menor amplitud de distribución en este eje, porque el agua es un medio que atenúa las diferencias de temperaturas.

En el análisis anterior, los cuadrantes del ordenamiento formado por los dos primeros ejes del análisis NMDS se pueden caracterizar como frío/seco el superior izquierdo, y frío/húmedo el superior derecho; por el contrario los cuadrantes inferiores representarían condiciones cálido/secas en el lado izquierdo, y cálido/húmedas, en el derecho.

La Fig. 7 muestra la distribución de los centroides de las comunidades vegetales estudiadas en el plano formado por los dos primeros ejes del análisis. La distribución de las asociaciones vegetales se corresponde perfectamente con la distribución de las especies (Fig. 6). En el centro del espacio multidimensional se presentan las dos praderas húmedas: Mentho-Juncetum microcephalii y Juncetum procerii; algo más retirado aparecen el espinal en el cuadrante inferior izquierdo y el Scirpetum californiae, en el inferior derecho. En el extremo derecho del primer eje se ubican el matorral terciario Rubo-Cestretum parqui y el bosque esclerófilo, Quillajo-Lithraetum causticae; entre este último y el espinal pero más distanciado hacia abajo, se presenta la plantación de pino. Nuevamente el espinal ocupa la posición intermedia en el gradiente de humedad entre las praderas húmedas y los bosques. En el extremo izquierdo del eje horizontal se ubican el matorral terciario de huatro (Gutierrezo-Baccharietum linearis) y el bosque de temo-pitra (Temo-Myrceugenietum exsuccae). Esta proximidad es difícil de explicar, a menos que el matorral de huatro se haya ubicado primitivamente en el ecotono entre el bosque pantanoso y el bosque esclerófilo, lo que deberá ser confirmado a futuro.

En el extremo derecho del primer eje horizontal se ubican las comunidades acuáticas Polygono-Ludwigietum peploidis, Utriculario-Ceratophylletum chilensis, Lemno-Azolletum filiculoidis y Potamogetetum lucentis; esta última comunidad, propia de aguas profundas, se separa un poco hacia abajo por el segundo eje. Con excepción de la plantación boscosa de pino y el espinal, el resto de las comunidades no se diferencian por

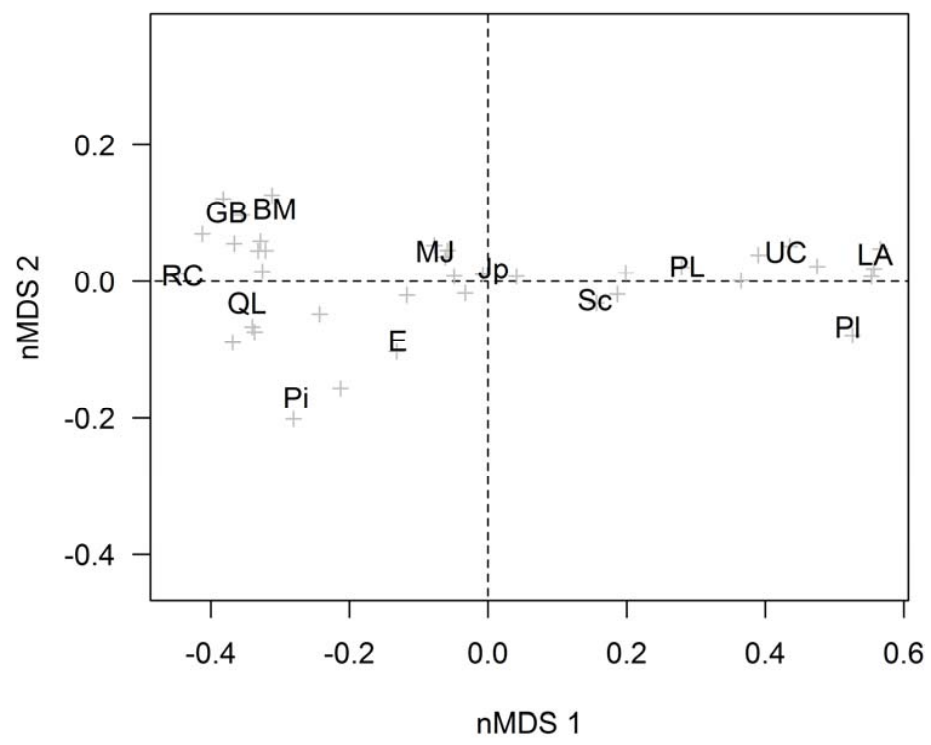

Fig. 7. Distribución de las asociaciones vegetales de la Tabla 2 en los dos primeros ejes del análisis nMDS. El significado de las abreviaturas de las asociaciones vegetales se encuentra en la Tabla 1. Las cruces corresponden a la ubicación de las especies.

Fig. 7. Distribution of the plant associations of Table 2 in the first two axes of nMDS analysis. The meaning of the abbreviations of plant associations found in Table 1. The crosses correspond to the location of the species. 
el segundo eje, demostrando que la temperatura no es un factor importante para la diferenciación de la vegetación de la laguna de las Ciénagas del Name, en comparación con la humedad.

\section{Relaciones dinámicas entre comunidades}

Con el resultado obtenido del análisis de los datos de la Tabla 2, con ayuda de la literatura disponible y con observaciones de terreno sobre la distribución espacial de las comunidades vegetales, se establecieron las posibles relaciones dinámicas de origen entre las diferentes comunidades. La dinámica de la vegetación presentaría dos series de sucesión, la primera de degradación de origen secundario y que va desde el bosque esclerófilo a las plantaciones, para luego llegar a estadios de degradación como el espinal y el matorral de huatro, y la segunda, de origen primario, que va desde la comunidad acuática sumergida de ceratofilo hasta el bosque nativo pantanoso de temo-pitra, que sería la comunidad terminal.

La primera es terrestre y representa la dinámica de degradación del primitivo bosque esclerófilo al ser talado, para disponer de suelos agrícolas que al degradarse son reemplazados por plantaciones de pino. Al talar el bosque y destinar el suelo al pastoreo se produce una degradación que va desde la formación del espinal de Acacia caven hasta un matorral de huatro que cubre los suelos altamente erosionados por el sobrepastoreo. Se supone que al cosechar la plantación de pino y abandonar los suelos, estos podrían ser también invadidos por el espinal, el cual eventualmente y a largo plazo, podría regenerar el bosque esclerófilo primitivo. En caso contrario, el destino final podría ser también la instalación de un matorral de huatro que por el estado de degradación en que se encuentra el suelo, no permitiría la regeneración del primitivo bosque esclerófilo.

La sucesión acuática primaria correspondería a un hidrosere natural que va rellenando la laguna, debido a la productividad de las plantas palustres y acuáticas, que acopian abundante necromasa en el sustrato, proceso que es acelerado por la sedimentación provocada por la erosión de las lomas adyacentes. Esta serie comenzaría en la comunidad sumergida de ceratofilo que ocupa el espejo de agua, y continuaría con la comunidad natante de clavito de agua, la que daría paso al totoral. Esta serie acuática finalizaría en un bosque pantanoso de temo-pitra. Cuando se tala el bosque pantanoso y se realiza pastoreo, se forman praderas húmedas que se suceden de acuerdo a la humedad del gradiente: primero la pradera de junquillo, luego la de poleo-junquillo rojo, para terminar en la pradera húmeda de junquillo rojo. Estas praderas se forman también al pastorear el pantano de totora. Las praderas húmedas men- cionadas están siendo invadidas por el espinal a medida que aumenta la sequía del suelo, por descenso del agua y aumento de la sedimentación. Estas praderas húmedas y el espinal de Acacia caven ponen en contacto las dinámicas de las dos series de vegetación descritas.

A la descripción anterior hay que agregar comunidades que representan desviaciones de la serie normal, el matorral de zarzamora $(R u-$ bo-Cestretum parqui) y las asociaciones acuáticas Lemno-Azolletum filiculoidis (comunidad flotante libre de flor del pato) y Potamogetetum lucentis (comunidad sumergida de huiro verde). El matorral de zarzamora es una comunidad terciaria que puede invadir terrenos alterados por el pastoreo, pero que en las Ciénagas del Name se forma en suelos ruderales de construcciones humanas, en este caso, caminos.

La comunidad flotante libre de flor del pato coloniza lugares protegidos del viento y generalmente muy contaminados por desechos orgánicos, y no tiene relación de continuidad ni de origen con las otras comunidades acuáticas. La comunidad sumergida de huiro verde ocupa el lugar de la comunidad de ceratofilo, en aguas profundas, y podría ser reemplazada por ésta al disminuir la profundidad del agua por relleno. Esta posibilidad deberá ser confirmada a futuro, ya que el análisis de los datos no lo demuestra, porque hay una mayor cercanía florística entre esta comunidad y la comunidad flotante libre del Lemno-Azolletum filiculoidis La dinámica vegetacional propuesta y que trata de reconstruir el curso de la evolución de los seres vegetacionales se representa gráficamente en un modelo conceptual, que permite apreciar estos resultados en forma resumida y comprensible (Fig. 8)

\section{DISCUSION}

Se confirma que los estudios vegetacionales con la metodología fitosociológica de la Escuela Sigmatista del Sur de Europa son previos y necesarios para conocer, delimitar y describir el mosaico vegetacional de un lugar (Dengler et al., 2008). Al clasificar y ordenar los sintaxa de este mosaico con método estadísticos multivariados es posible comprender las relaciones de origen entre las comunidades, y establecer así, los factores naturales y antrópicos que podrían haber causado cambios en la vegetación (Alarcón, 2014).

Lo anterior expresado en un modelo conceptual, permite predecir los cambios en la vegetación ante un determinado impacto, y conocer los impactos que actuaron en el pasado sobre ella. Esto permite planificar el uso del suelo e intervenir en el sistema, aplicando medidas que permitan direccionar el desarrollo de la vegetación, 


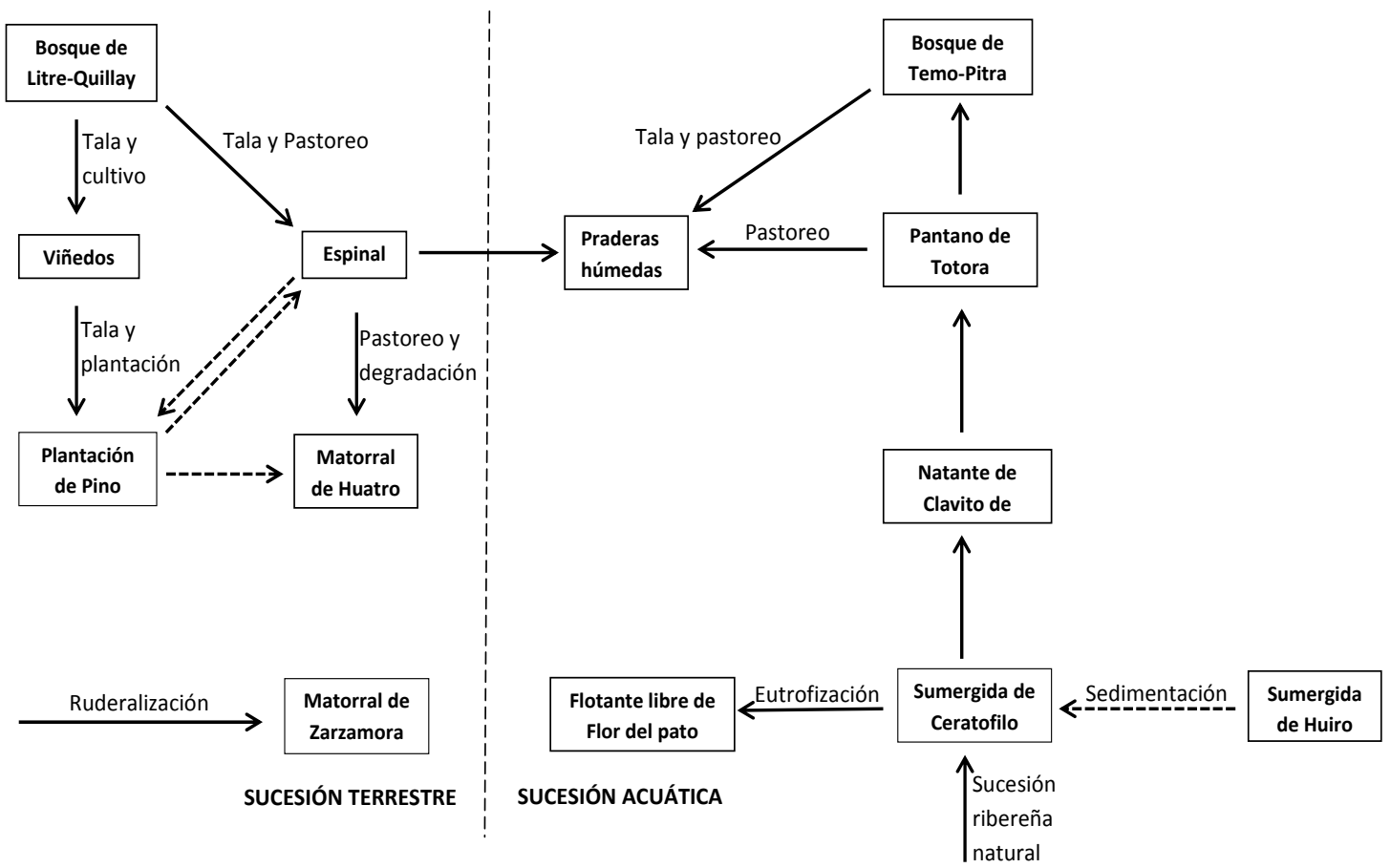

Fig. 8. Modelo conceptual de las probables relaciones de origen entre las asociaciones vegetales acuáticas presentes en las Ciénagas del Name y terrestres de las zonas aledañas (terrestres). Las flechas enteras indican relaciones de origen con alta y las punteadas con baja probabilidad. Sobre las flechas se indica el o los factores que provocarían el cambio antrópico de una comunidad a otra.

Fig. 8. Conceptual model of the likely origin relationships between aquatic plant associations in "Ciénagas del Name" and terrestrial plant associations in surrounding areas. The full arrows indicate relations of origin dotted with high and low probability. Above the arrows indicates the factors that provoke the anthropic change from one community to another.

favoreciendo determinados manejos, facilitando la regeneración de la misma y la conservación de los hábitats tan necesarios para la mantención de la biodiversidad, especialmente de avifauna (Estades et al. 2012).

La vegetación palustre que primitivamente limitaba con bosques nativos ha sido fuertemente influenciada por el pastoreo, que por la baja capacidad de carga de los suelos, cambia la compactación del sustrato y su relieve (Ramírez y Álvarez, 2012). Estos cambios permiten la formación de praderas húmedas antropogénicas que pueden ser colonizadas por Acacia caven, permitiendo posiblemente a futuro, la recuperación del bosque esclerófilo (Ramírez et al., 2014b). Esta especie actúa como una entidad pionera, lo que cambia el concepto predominante, en el sentido de que ella sería sólo una indicadora de la degradación del bosque esclerófilo y de su matorral secundario de reemplazo que se detiene en la etapa de espinal (Balduzzi et al, 1982). Acacia caven demuestra una gran amplitud ecológica, apareciendo en un mayor número de comunidades, poniendo en con- tacto la serie acuática natural y aquella terrestre degradada por la actividad humana, lo que también explica su amplia distribución geográfica en Chile (Ramírez et al., 2014b).

Aunque comúnmente el sotobosque de las plantaciones forestales está dominado por malezas tanto nativas como introducidas, en las Ciénagas del Name sirve de refugio a varias especies leñosas nativas, lo que a futuro podría ser utilizado para la recuperación del bosque original, siempre que la cosecha de las plantaciones se haga con poco impacto. Incluso, en estas plantaciones forestales hay una recuperación del suelo, que se cubre de un horizonte orgánico muy rico en actividad biológica, especialmente micorrícica (Ramírez et al., 2014b).

En la vegetación acuática se conservan varias especies nativas, dada la condición de azonalidad del biótopo, aunque el mismo factor agua, puede limitar la riqueza específica de las comunidades vegetales terrestres (Walter, 1997). La acción antrópica mediante pastoreo y cultivo es más efectiva sobre la vegetación zonal terrestre 
alterando considerablemente las condiciones originales (Ellies y Ramírez, 1994).

Uno de los factores más influyentes sobre la vegetación del lugar estudiado resultó ser el agua o la humedad del suelo, la cual aumenta hacia el centro de la laguna y se retira en esa misma dirección hacia la ribera. Este proceso de relleno de la laguna, provocado tanto por el efecto natural de la alta producción orgánica de las comunidades acuáticas y palustres, se ve acrecentado por la erosión de las tierras aledañas, actualmente sobrepastoreadas y labradas. Este fenómeno podría aumentar con el cambio climático, que también incrementaría la evapotranspiración de las plantas. En este caso, al secarse las riberas, aumenta la degradación biológica de la materia orgánica del suelo y se libera el carbono almacenado en ella (Arellano et al., 2013, Ramírez et al., 2014c).

\section{RECONOCIMIENTOS}

Investigación financiada parcialmente por Empresas Arauco mediante el Proyecto "Monitoreo Humedal del Name" convenio PUC-ARAUCO y por el Proyecto FONDECYT 1141117 a J.M. Fariña.

\section{LITERATURA CITADA}

Alarcón, W. 2014. Flora, vegetación y hábitats del humedal "Millahuillin" en Máfil, provincia de Valdivia, Región de los Ríos, Chile". 70 p. Trabajo de titulación. Escuela Ingeniería en Conservación de Recursos Naturales, Universidad Austral de Chile, Valdivia, Chile.

Amigo, J., and L. Flores-Toro. 2013. A new contribution to syntaxonomy of sclerophyllous forest and pre-forest of Central Chile: Lithraeion causticae alliance. International Journal of Geobotanical Reseach 3:47-67.

Arellano, E., F. Meza, M. Miranda, y A. Camaño. 2013. El cuidado de los humedales y su rol en el secuestro de carbono. Agronomía y Forestal 47:22-27.

Balduzzi, A., I. Serey, R. Tomaselli, and R. Villaseñor. 1982. Degradation of the mediterranean type of vegetation in Central Chile. Ecología Mediterranea 7(1/2):223-240.

Bray, J.R., and J.T. Curtis. 1957. An ordination of the upland forest communities of Southern Wisconsin. Ecological Monographies 27:325340.

Clarke, K.R. 1993. A method of linking multivariate community structure to environmental variables. Marine Ecology Progress Series 92:205-219.
Dengler, J., M. Chytry, and J. Ewald. 2008. Phytosociology. p. 2767-2779. In S.E. Jørgensen, y B. D. Fath (eds.) Encyclopedia of Ecology. Elsevier, Oxford, England.

Ellies, A., y C. Ramírez. 1994. Efecto del manejo sobre la estructura del suelo y la biodiversidad específica vegetal. p. 79-103. En Seminario Medio Ambiente, Biodiversidad y Actividades Productivas. 14-15 de junio de 1994. Instituto Forestal, Universidad de Chile, Santiago, Chile.

Estades, C., M. Vukasovic, y J. Aguirre. 2012. Aves en los humedales costeros de Chile. p. 67-99. En J.M. Fariña y A. Camaño (eds.) Humedales costeros de Chile: Aportes científicos a su gestión sustentable. Ediciones Universidad Católica de Chile, Santiago, Chile.

Gautreau, P., y F. Lezama. 2009. Clasificación florística de los bosques y arbustales de las sierras de Uruguay. Ecología Austral 19:81-92.

Greca, I.M., y M.A. Moreira. 1998. Modelos mentales, modelos conceptuales y modernización. Cad. Cat. Ens. Fis. 15(2):107-120.

Legendre P., and L. Legendre. 1998. Numerical ecology. 853 p. Elsevier, Amsterdam, The Netherlands.

Mueller-Dombois, D., and H. Ellenberg. 1974. Aims and methods of vegetation ecology. 547 p. Wiley \& Sons, New York, USA.

Ortuño, T., S. Beck, y L. Sarmiento. 2006. Dinámica sucesional de la vegetación en un sistema agrícola con descanso largo en el Altiplano central boliviano. Ecología en Bolivia 41(3):40-70.

Peña-Cortes, F., G. Rebolledo, K. Hermosilla, E. Hauenstein, C. Bertran, R. Schlatter, y J. Tapia. 2006. Dinámica del paisaje para el período 1980-2004 en la cuenca costera del Lago Budi, Chile. Consideraciones para la conservación de sus humedales. Ecología Austral 16:183-196.

R Development Core Team. 2008. R: A language and environment for statistical computing. $\mathrm{R}$ Foundation for Statistical Computing. ISBN 3-900051-07-0. Vienna, Austria. URL http:// www.R-project.org.

Ramírez, C., y M. Álvarez. 2012. Flora y vegetación hidrófila de los humedales costeros de Chile. p. 101-145. En J.M. Fariña y A. Camaño (eds.) Humedales costeros de Chile: Aportes científicos a su gestión sustentable. Ediciones Universidad Católica de Chile, Santiago, Chile.

Ramírez, C., J. Barrera, D. Contreras, y J. San Martín. 1988. Estructura y regeneración del matorral de Ulex europaeus en Valdivia, Chile. Medio Ambiente (Valdivia) 9(1):143-149. 
Ramírez, C., V. Sandoval, C. San Martín. M. Álvarez, Y. Pérez, y C. Novoa. 2012. El paisaje rural antropogénico de Aisén, Chile: Estructura y dinámica de la vegetación. Gayana Botanica 69(2):219-231.

Ramírez, C., J.M. Fariña, D. Contreras, A. Camaño, C. San Martín, M. Molina, et al. 2014a. La diversidad florística del humedal "Ciénagas del Name" (Región del Maule) comparada con otros humedales costeros de Chile Central. Gayana Botanica 71(1):59-70.

Ramírez, C., J.M. Fariña, A. Camaño, D. Contreras, C. San Martín, J. Varas, et al. 2014b. Estructura y clasificación de la vegetación potencial y actual del humedal "Ciénagas del Name" en Chile Central: un estudio de la oferta de hábitats. Chilean Journal of Agricultural \& Animal Sciences (ex Agro-Ciencia) 30(1):29-44.

Ramirez, C., C. San Martín, J.M. Fariña, A. Camaño, M. Álvarez, Y. Pérez, et al. 2014c. Humedales costeros de la Región del Biobío (Chile): Un gradiente de vegetación y una nueva asociación vegetal de marisma. Chilean Journal of. Agricultural \& Animal Sciences (ex Agro-Ciencia) 30 (3):233-246.
Roeland K., and R. Coe. 2005. Tree diversity analysis. A manual and software for common statistical methods for ecological and biodiversity studies. World Agroforestry Centre (ICRAF), Nairobi. Kenya.

San Martín, J., y C. Donoso. 1996. Estructura florística e impacto antrópico en el bosque maulino de Chile. p. 153-168. En J. Armesto, C. Villagrán y M. Kalin-Arroyo (eds.) Ecología de los bosques nativos de Chile. Editorial Universitaria, Santiago, Chile.

San Martín, C., V. Sandoval, M. Álvarez, O. Vidal, Y. Pérez, y J.L. Solís. 2014. Comparación de etapas de degradación vegetacional con manejo pecuario utilizando valores bioindicadores de Ellenberg en la Patagonia Chile. Bosque 35(2):141-154.

Venables W.N., and B.D. Ripley. 2002. Modern applied statistics with S. 3348 p. Missouri Botanical Garden Press, Missouri, USA.

Walter, H. 1997. Zonas de vegetación y clima. 245 p. Ediciones Omega, Barcelona, España. 